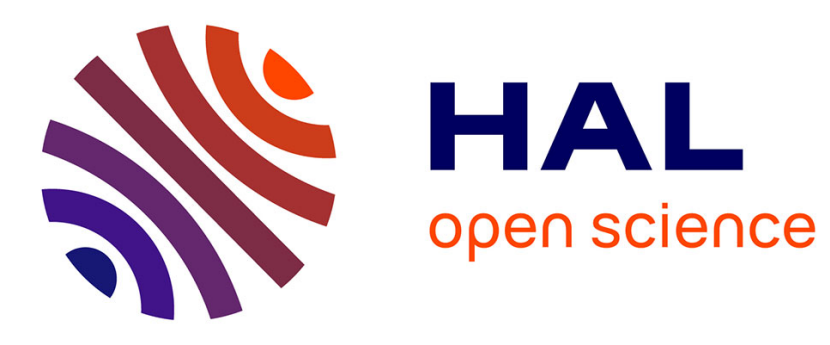

\title{
Exploitation des mesures de diffusion de neutrons polarisés
}

\author{
R. Sené, J. Kahane, P. Delpierre, M. Heyman
}

\section{To cite this version:}

R. Sené, J. Kahane, P. Delpierre, M. Heyman. Exploitation des mesures de diffusion de neutrons polarisés. Revue de Physique Appliquée, 1969, 4 (2), pp.115-115. 10.1051/rphysap:0196900402011500 . jpa-00243170

\section{HAL Id: jpa-00243170 https://hal.science/jpa-00243170}

Submitted on 1 Jan 1969

HAL is a multi-disciplinary open access archive for the deposit and dissemination of scientific research documents, whether they are published or not. The documents may come from teaching and research institutions in France or abroad, or from public or private research centers.
L'archive ouverte pluridisciplinaire HAL, est destinée au dépôt et à la diffusion de documents scientifiques de niveau recherche, publiés ou non, émanant des établissements d'enseignement et de recherche français ou étrangers, des laboratoires publics ou privés. 


\title{
EXPLOITATION DES MESURES DE DIFFUSION DE NEUTRONS POLARISÉS
}

\author{
R. SENÉ, J. KAHANE, P. DELPIERRE et M. HEYMAN, \\ Physique Atomique et Moléculaire, Collège de France.
}

\begin{abstract}
Résumé. - Nous décrivons une méthode d'exploitation en différé d'un grand nombre de spectres élémentaires avant leur interprétation.

Abstract. - We describe an elaborated method for the delayed use of a large number of elementary spectra before their interprétation.
\end{abstract}

Les mesures d'asymétrie dans la diffusion des neutrons de $14 \mathrm{MeV}$ polarisés effectuées avec le générateur du Collège de France sont conduites, faute de disposer d'un système élaboré d'acquisition des données, de la façon suivante. Nous décomposons le temps d'enregistrement en périodes élémentaires (60 ou $80 \mathrm{mn}$ pour avoir un contrôle suffisant) pour lesquelles nous enregistrons : i) les 8 spectres de temps-de-vol (8 voies de détection simultanées) [1]; ii) les données de comptage : nombre d'alpha, nombre de particules au-dessus du seuil détectées sur chaque voie; iii) les données «générateur » permettant de connaître la polarisation des particules incidentes : anisotropie à la production, comptages polarimètre [2]. Enregistrées sur un support compatible, ces données sont transférées à l'aide de l'ordinateur du laboratoire sur une bande magnétique grâce à laquelle le traitement est effectué plus aisément. Un programme gère l'ensemble des opérations portant pour la dernière expérience sur environ 2000 spectres.

La durée de chaque groupe de mesures étant de 100 à 200 heures, nous avons dû corriger l'effet de certaines dérives instrumentales. Le premier de ces traitements corrige les dérives intervenant dans la chaîne d'analyse du temps-de-vol. Les dérives du convertisseur tempsamplitude et du convertisseur analogique-digital donnent après totalisation un lissage des spectres. Après repérage des pics sur chaque spectre, un recentrage est effectué par une homothétie (dérive du gain) et par une translation (dérive du seuil) ( $f g .1$ ). Le second traitement est un calcul du niveau de bruit de fond. Celui-ci est produit par les coïncidences fortuites dont nous pouvons calculer le niveau sur chaque spectre en considérant que chaque canal est une coïncidence dont la résolution est la largeur en temps du canal.

La normalisation en polarisation incidente est faite spectre par spectre avant la totalisation. Les séparations de pics non résolus sont obtenues par ajustement de distributions. Nous avons aussi ajusté un niveau de bruit de fond sur des spectres non corrigés. L'accord

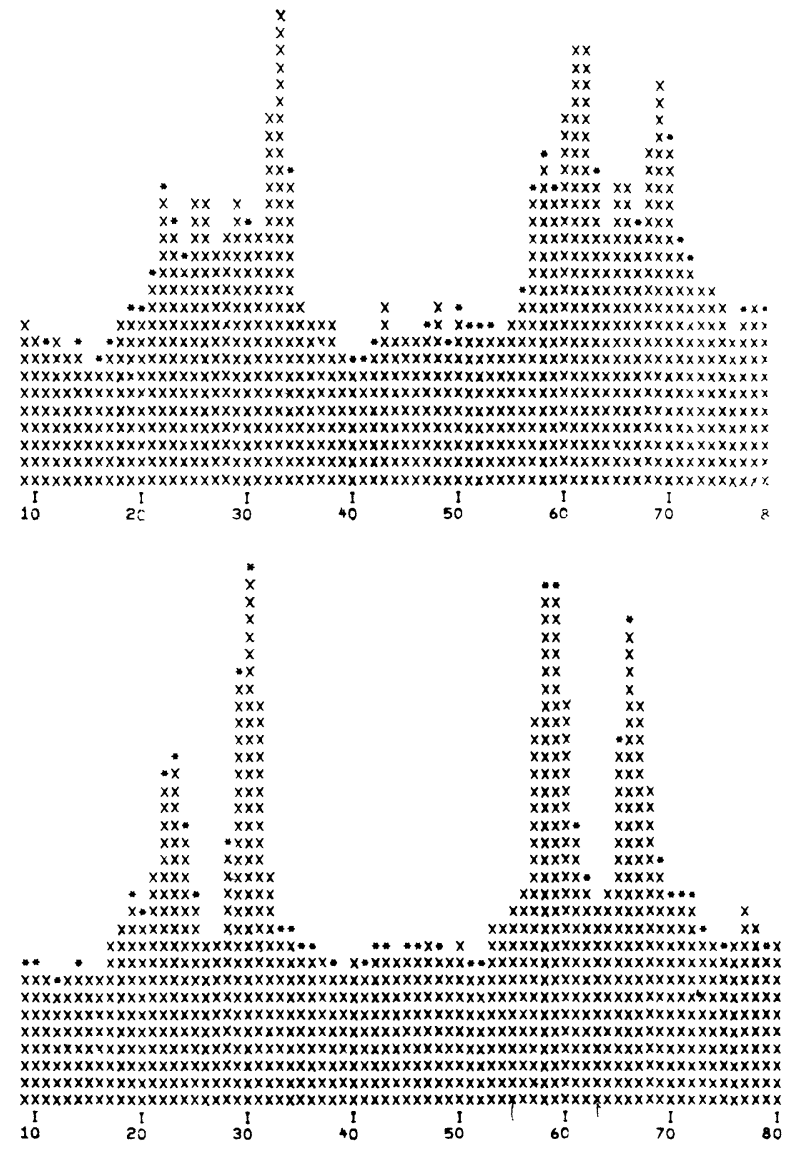

FIG. 1. - Spectres totalisés avant et après recentrage.

entre les deux méthodes est bon. Ces deux façons de procéder ont l'avantage de fixer le niveau de bruit de fond d'une manière objective. Les corrections de diffusion multiple sont effectuées en utilisant un programme de calcul de Joseph [3].

\section{BIBLIOGRAPHIE}

[1] SENÉ (R.) et al., ce colloque.

[2] DELPIERRE (P.) et al., ce colloque.

[3] JoSEPH, Thèse de doctorat, Lausanne, 1966. 